\title{
ANEURISMA DISSECANTE DE ARTÉRIA VERTEBRAL INTRACRANIANA FENESTRADA SUBMETIDO AO TRATAMENTO ENDOVASCULAR
}

\section{Relato de caso}

\author{
Guilherme Cabral de Andrade', Jean Gonçalves de Oliveira1, \\ Rafi Felício Bauab Dauar², Darcio Roberto Nalli³, Fernando Menezes Braga ${ }^{4}$
}

\begin{abstract}
RESUMO - Aneurismas dissecantes da artéria vertebral em seu segmento intracraniano são lesões pouco comuns, principalmente quando associadas a hemorragia subaracnóidea, sendo também raras as fenestrações da artéria vertebral. Apresentam elevada morbidade e mortalidade, com elevado índice de ressangramento e dificuldade de abordagem cirúrgica. Apresentamos o caso de um homem de 19 anos, o qual foi vitima de agressão física em região occipto-cervical, apresentando hemorragia subaracnóidea e aneurisma dissecante na artéria vertebral direita, a qual era fenestrada, sendo submetido ao tratamento endovascular. Realizamos revisão da literatura sobre o assunto, sendo colocado o tratamento endovascular como uma opção terapêutica para estes casos.
\end{abstract}

PALAVRAS-CHAVE: aneurisma dissecante, hemorragia subaracnóidea, artéria vertebral.

\begin{abstract}
Dissecting: aneurysm of the intracranial fenestrated vertebral artery submited to endovascular treatment case report

ABSTRACT - Dissecting aneurysms of the vertebral artery at its intracranial segment are uncommon lesions, mainly when associated to subarachnoid hemorrhage, being also rare fenestrations of the vertebral artery. They present high morbidity and mortality, with high rebleeding rate and difficulty of surgical approach. We present a 19 years old man who was victim of physical aggression in the occipto-cervical region, presenting subarachnoid hemorrhage and a dissecting aneurysm of the right vertebral artery, which had a fenestration, being submitted to endovascular treatment. We accomplished a literature review about this subject, proposing endovascular treatment as a therapeutic option for these cases.
\end{abstract}

KEY WORDS: dissecting aneurysm, subarachnoid hemorrhage, vertebral artery.

Aneurismas dissecantes da artéria vertebral em seu segmento intracraniano são lesões pouco comuns, tendo como principal conseqüência acidente vascular cerebral da circulação posterior com isquemias em tronco cerebral e hemisférios cerebelares ${ }^{1,2}$, sendo a síndrome de Wallenberg a apresentação clínica mais comumente encontrada nesses casos, com incidência que varia entre $26 \%$ e $43 \%^{3}$. A associação de dissecção aneurismática com hemorragia subaracnóidea (HSA) é ocorrência ainda menos comum ${ }^{1,2,4,5}$, com freqüência de $4,5 \%$ de todas as HSA, que possuem alto índice de ressangramento nas primeiras $24 \mathrm{~h}$, em torno de $24 \%$ e $30 \%$ e também de mortalidade elevada de $45 \%{ }^{2,6}$. O primeiro relato de lesão dissecante arterial é do ano de $1915^{7}$.Em revisão da literatura de 1924 a $1983^{1}$, foi observado HSA através de punção lombar em apenas 7 dos 36 casos, não havendo referência de traumatismo em nenhum destes. Outros autores relatam, com freqüência pouco maior, casos de dissecção da artéria vertebral associado com HSA ${ }^{2-6,8-13}$, embora não haja, em nenhum deles, associação com traumatismo crânio-cervical. Do ponto de vista angiográfico, essas lesões passaram a ser identificadas a partir de 1959 através de sinais como: retenção de meio de contraste $^{14}$, sinal do duplo lúmen ${ }^{15}$, sinal da roseta ${ }^{16} \mathrm{e}$ sinal do barbante ("string sign") ${ }^{17}$. Os casos de fenestração do sistema vértebro-basilar são raros e possuem incidência de $0,03 \%$ a $2,20 \%{ }^{18}$ e a sua associação com aneurisma sacular e dissecante é extrema-

Disciplina de Neurocirurgia do Hospital São Paulo (HSP) da Universidade Federal de São Paulo -Escola Paulista de Medicina (UNIFESP/EPM), São Paulo SP, Brasil e Hospital Estadual de Diadema, Diadema SP, Brasil (HED): ${ }^{1}$ Neurocirurgião pós-graduando em Neurocirurgia pela UNIFESP/EPM; ${ }^{2}$ Neurocirurgião do (HED); ${ }^{3}$ Neurorradiologista Chefe do Serviço de Neurorradiologia do HSP; ${ }^{4}$ Professor Titular da Disciplina de Neurocirurgia da UNIFESP/EPM.

Recebido 5 Julho 2004, recebido na forma final 4 Outubro 2004. Aceito 22 Novembro 2004.

Dr. Jean Gonçalves de Oliveira - Rua Sena Madureira 1123/61 - 04021-051 São Paulo SP - Brasil. E-mail: jeango/@uol.com.br 
mente rara ${ }^{19}$. A fisiopatologia é dada por falência embriológica, na involução dos canais de interconexão vascular presentes no $7^{\circ}$ segmento cervical arterial, o que resulta em duplicação e/ou fenestração da artéria vertebral ocorrido entre o $32^{\circ}$ e $40^{\circ}$ dia de gestação.

Relatamos caso submetido a cirurgia endovascular.

\section{CASO}

Homem de 19 anos, foi admitido no Pronto Socorro do Hospital Estadual de Diadema em abril de 2002, com quadro de cefaléia intensa após ter sido vitima de agressão física durante tentativa de assalto, com traumatismo direto em região occipto cervical com cabo de revólver, sem que houvesse perda de consciência. Logo após o trauma sentiu cefaléia intensa occipto-cervical e dor nucal. Ao ser admitido queixava-se de cefaléia holocraniana de forte intensidade. Apresentava-se consciente, orientado, com 15 pontos na escala de coma de Glasgow, sem apresentar déficits neurológicos focais, porém com rigidez nucal importante (+++/4+). Realizou exame de tomografia computadorizada de crânio (Fig 1A e 1B) que evidenciou HSA com classificação de Fisher grau III, além de hidrocefalia incipiente. Recebeu tratamento para HSA, realizando em seguida exame de angiografia digital cerebral, a qual se mostrava sem alterações vasculares na circulação anterior, porém com achados importantes na circulação vértebro-basilar, com artéria vertebral direita fenestrada e sinais angiográficos de dissecção arterial como o sinal da pérola e barbante, ("pearl and string sign") (Fig 2A e 2B).

Em virtude dos achados angiográficos que mostravam a manutenção da patência da artéria vertebral direita pelo outro ramo da fenestração e ausência de alterações na

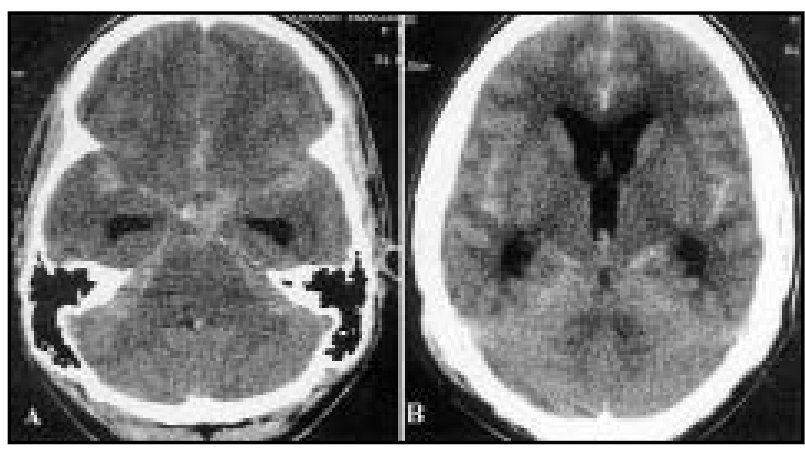

Fig 1-A e 1-B. Tomografia computadorizada de crânio evidenciando hemorragia subaracnóidea com Fisher III e hidrocefalia incipiente.

artéria vertebral contralateral, optou-se pelo tratamento endovascular, com o uso de micromolas ("microcoils") com oclusão do ramo da fenestração no qual se encontrava o aneurisma dissecante (Fig 3A). Foi possível visualizar a permeabilidade do sistema vértebro-basilar, com o enchimento de todo o sistema através das duas artérias vertebrais e oclusão do ramo da artéria vertebral direita onde ocorreu a dissecção (Fig 3B e 3C). O paciente recebeu alta hospitalar no $3^{\circ}$ dia de pós-operatório, assintomático e sem apresentar déficits neurológicos.

\section{DISCUSSÃO}

As artérias intracranianas, assim como as artérias do canal vertebral, possuem diferenças anatômicas quando comparadas às artérias de diâmetro similar no restante do corpo. Estas consistem em: redução das camadas adventícia e média pela diminuição da quantidade de fibras elásticas, além de possuírem fibras colágenas mais finas e pela ausência da

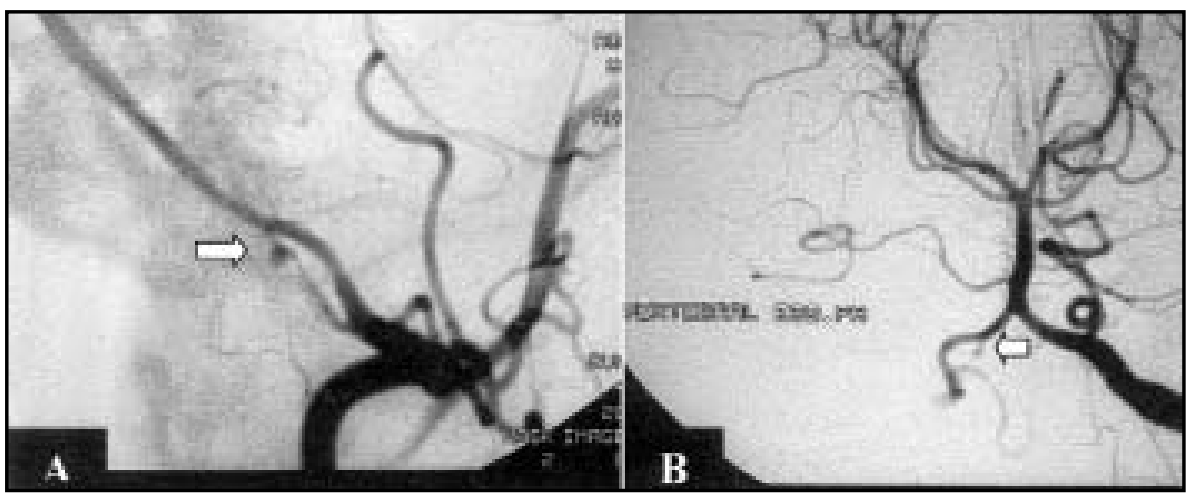

Fig 2-A e 2-B. Angiografia digital que mostra artéria vertebral direita fenestrada e dissecção em um dos ramos da fenestração com um aneurisma dissecante e sinal angiográfico "pearl and string sign".

Fig 3-A, B e C. Imagem radiográfica mostrando a colocação das molas "coils"; 3-B e 3-C. Controle angiográfico perioperatório mostrando oclusão completa do ramo onde houve a dissecção.

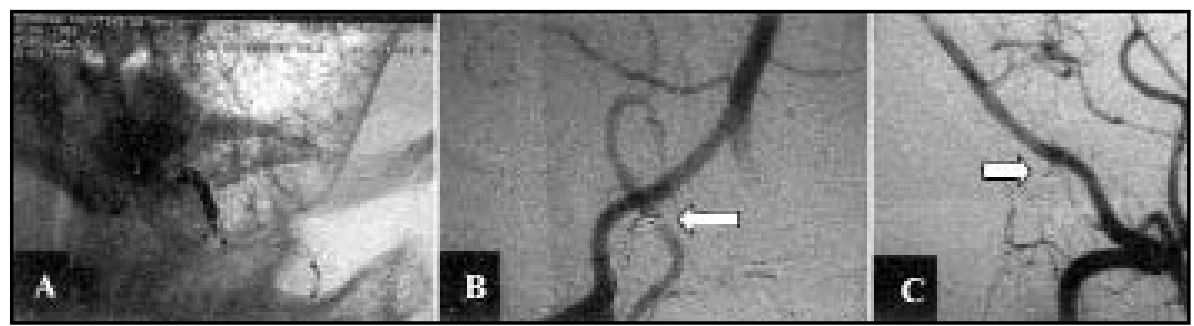




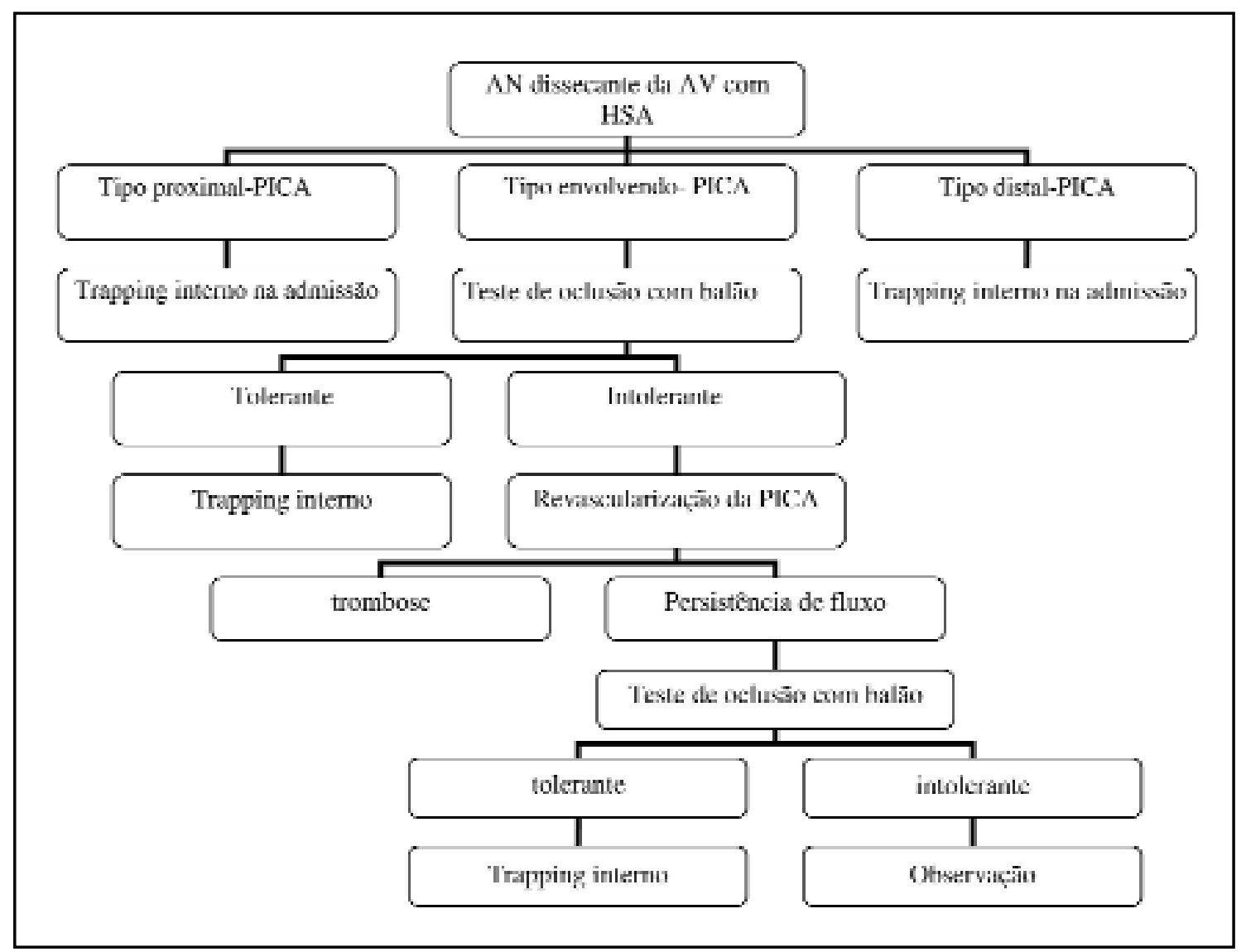

Fig 4. Organograma representando proposta de manejo dos aneurismas dissecantes da artéria vertebral que evoluem com hemorragia subaracnóidea. (An, aneurisma; PICA, artéria cerebelar póstero-inferior; HAS, hemorragia subaracnóidea) (Lihara 2002).

camada elástica externa ${ }^{20}$. Estas diferenças ocorrem desde $0,5 \mathrm{~cm}$ do segmento extradural há até $0,5 \mathrm{~cm}$ do segmento intradural da artéria vertebral ${ }^{21}$ e que devem ser consideradas para definição da abordagem desses aneurismas. Na quase totalidade das dissecções das artérias vertebrais o hematoma intramural forma-se entre a lâmina elástica interna e a camada média. Raramente a dissecção envolve a camada subadventícia e, quando isto acontece, pode ocorrer ruptura para o espaço subaracnoideo resultando em HSA ${ }^{2,4-13}$. Segundo Yamaura ${ }^{10}, 28 \%$ dos aneurismas da circulação posterior, que evoluem com HSA, são lesões aneurismáticas dissecantes.

Em relação aos aneurismas dissecantes da artéria vertebral, encontramos 4 classificações na literatura. A primeira, de Sasaki ${ }^{11}$, classifica em dois grupos: grupo 1- dissecção confinada à artéria vertebral e grupo 2- com extensão para a artéria basilar; esta classificação tem importância apenas para o prognóstico da lesão. Uma segunda classificação, de Sano ${ }^{12}$, divide em: grupo de aneurismas com dissecção unilateral e grupo de aneurismas com dissecção cir- cunferencial. Uma terceira classificação divide em dois tipos: 1- do tipo com uma única entrada (entry only type), onde existe apenas um orifício de entrada no pseudo lúmen; ocorre em maior número e possui curso clínico instável com HSA e infarto cerebral; e 2-do tipo entrada e saída (entry exit type), com orifício de entrada e saída, possuindo fluxo sanguíneo constante no interior do pseudo lúmen e com maior estabilidade hemodinâmica ${ }^{13}$. Uma quarta e mais recente classificação subdivide estes aneurismas em 3 grupos: grupo dos aneurismas proximais à PICA (artéria cerebelar póstero-inferior), grupo dos aneurismas envolvendo a PICA e um terceiro grupo dos aneurismas distais à PICA (Fig 4); esta classificação segue um organograma para a definição da conduta a ser tomada, tendo como referência o tratamento endovascular ${ }^{4}$.

As causas de dissecções são as mais variáveis, indo desde as dissecções espontâneas em pacientes portadores de migrânea ${ }^{22}$, displasia fibromuscular ${ }^{23}$, necrose cística medial ${ }^{24}$, síndrome de Marfan ${ }^{25}$, arterite ${ }^{26}$ assim como causas traumáticas que podem 
ocorrer por lesões mínimas ou triviais como durante atividade de esporte, espirros, dormir em posições não usuais, rotação da cabeça, ou mesmo em virtude de torções excessivas da coluna cervical durante ressucitação cardio-respiratória, entubação orotraqueal e durante crises convulsivas tônico-clônicas ${ }^{27}$.

A apresentação clínica mais comumente encontrada nos aneurismas dissecantes da artéria vertebral intracraniana é a dor occipto-cervical, a qual precede a cefaléia ou inicia-se simultaneamente a ela, sendo ipsolateral à dissecção e atingindo cerca de $75 \%$ dos pacientes ${ }^{28}$. Porém, em muitos casos, o diagnóstico não é suspeitado por existir grande variabilidade de sinais e sintomas, os quais incluem: locked-in síndrome, síndrome de Wallemberg, infarto medular uni ou bilateral, síndrome cerebelar, síndrome vestibular, amnésia transitória, tinitus, turvação visual, hemianopsia, isquemia transitória vértebro-basilar e $\mathrm{SH}^{27}$. O diagnóstico radiológico é definido com a realização da angiografia das artérias vertebrais, porém a ressonância magnética e a angioressonância de crânio são usadas como triagem, sendo valorizado por não ser método invasivo e possuir alta resolução na fossa posterior, a presença de artefatos ósseos para a visualização de trombos intramurais e da ausência de sinal de fluxo arterial "flow void" 29 . Entretanto, a angiografia digital é o método de escolha para o diagnóstico e para que se defina a conduta terapêutica.

A abordagem com ligadura da artéria proximal ao aneurisma, método descrito por Hunter ${ }^{30}$, que promove diminuição da pressão e fluxo no interior do aneurisma e conseqüente trombose, tem sido ainda uma opção de tratamento para alguns autores, embora possua risco de ressangramento em virtude da manutenção do fluxo retrógrado da artéria vertebral distal e de seus ramos, limitando a progressão do trombo presente no local de dissecção do aneurisma. Há ainda outras opções de tratamento cirúrgico como: isolamento segmentar "trapping", envolvimento "wrapping" e clipagem direta do aneurisma'. Devido à sua localização, sendo a maioria dos aneurismas distal à PICA e próximo à linha média, há dificuldade de abordagem cirúrgica, havendo aumento da morbidade por paralisia de nervos cranianos baixos. Ainda como complicação intraoperatória, a ruptura do aneurisma pode ocorrer espontaneamente ou por manipulação, em virtude da espessura adelgaçada da camada adventícia ${ }^{10}$.

O tratamento cirúrgico por abordagem endovascular tem sido uma opção recentemente utilizada para estes aneurismas, através de técnicas endovasculares com uso de balões destacáveis, angioplastia transluminal, e oclusão (ou "trapping" interno) com molas (coils) 4,6,8,10. A classificação destes aneurismas de acordo com a proximidade da PICA, fez com que Lihara ${ }^{4}$ definisse a estratégia de abordagem endovascular, realizando oclusão interna com coils, na admissão, nos casos em que a dissecção é proximal ou distal à PICA. Quando há o envolvimento da PICA no segmento dissecado, segue-se um algorritimo em que se utiliza o teste de oclusão com balão para verificação da manutenção de fluxo através da PICA e determinação da conduta terapêutica (Fig 4).

Aneurisma dissecante da artéria vertebral em seu segmento intracraniano é patologia infrequente, principalmente quando associada a HSA. O caso apresentado possui algumas peculiaridades por apresentar uma alteração anatômica rara (fenestração da artéria vertebral) e um aneurisma dissecante em um dos ramos da fenestração, o qual teve como fator causal um traumatismo occipto-cervical. Devemos sempre ter como possibilidade de diagnóstico diferencial da HSA caudada por aneurisma sacular, a probabilidade de um aneurisma dissecante, sendo de fundamental importância a realização da angiografia digital, sempre com a verificação dos 4 vasos, nos casos de HSA. No caso de aneurisma dissecante da artéria vertebral, propomos a abordagem endovascular como primeira opção de tratamento, seguindo o algorritimo referido.

\section{REFERÊNCIAS}

1. Berger MS, Wilson CB. Intracranial dissecting aneurysms of the posterior circulation. J Neurosurg 1984;61:882-894.

2. Yamaura A, Watanabe Y, Saeki N. Dissecting aneurysms of the intracranial vertebral artery. J Neurosurg 1990;72:183-188.

3. Hosoya T, Watanabe N, Yamaguchi K, Kubota H, Onodera Y. Intracranial vertebral artery dissection in Wallenberg syndrome. Am J Neuroradiol 1994;15:1161-1165.

4. Lihara K, Sakai N, Murao K, et al. Dissecting aneurysms of the vertebral artery: a management strategy. J Neurosurg 2002;97:259-267.

5. Yasui T, Komiyama M, Nishikawa M, Nakajima H. Subarachnoid hemorrhage from vertebral artery dissecting aneurysms involving the origin of the posteroinferior cerebellar artery: report of two cases and review of the literature. Neurosurgery 2000;46:196-200.

6. Coley SC, Clifton A. Dissecting vertebral artery aneurysm: diagnosis and coil embolization. Case report. Br J Radiol 1999;72:408-411.

7. Turnbull HM. Alterations in arterial structure, and their relation to syphilis. QJM 1915;8:201-254.

8. Halbach VV, Higashida RT, Dowd CF, et al. Endovascular treatment of vertebral artery dissections and pseudoaneurysms. J Neurosurg 1993; 79:183-191.

9. Yasui $\mathrm{T}$, Sakamoto $\mathrm{H}$, Kishi $\mathrm{H}$, et al. Bilateral dissecting aneurysms of the vertebral arteries resulting in subarachnoid hemorrhage: case report. Neurosurgery 1998;42:162-165.

10. Yamaura I, Tani E, Yokota M, et al. Endovascular treatment of ruptured dissecting aneurysms aimed at occlusion of the dissected site by using Guglielmi detachable coils. J Neurosurg 1999;90:853-856.

11. Sasaki O, Ogawa H,Koike T, Koizume T, Tanaka R, A clinicopathological study of dissecting aneurysms of the intracranial vertebral artery. J Neurosurg 1991;75:874-882. 
12. Sano H, Kato $\mathrm{Y}, \mathrm{Okuma}$ I, et al. Classification and treatment of vertebral dissecting aneurysm. Surg Neurol 1997;48:598-605.

13. Mizutani T,Kojima H,Asamoto S, Yoshimasa M.Pathological maechanism and three-dimensional structure of cerebral aneurysms.J Neurosurg 2001;94:712-717.

14. Anderson RM, Schechter MM. A case of spontaneous dissecting aneurysm of the internal carotid artery. J Neurol Neurosurg Pyschiatry 1959;22:195-201.

15. Kunze S, Schefer W. Angiographic demonstration of a dissecting aneurysm of the middle cerebral artery. Neuroradiology 1981;2:201-206.

16. Scott GE, Neubuerger KT, Denst J. Dissecting aneurysm of intracranial arteries. Neurology 1960;10:22-27.

17. Ojemann RG, Fischer CM, Rich JC. Spontaneous dissecting aneurysm of the internal carotid artery. Stroke 1972;3:434-440.

18. Kowada M, Yamaguchi K, Takahashi H. Fenestration of the vertebral artery with a review of 23 cases in Japan. Radiology 1972;103:343-346.

19. Zhang QJ, Kobayashi S, Gibo H, Hongo K. Vertebrobasilar junction fenestration associated with dissecting aneurysm of intracranial vertebral artery. Stroke 1994;25:1273-1275.

20. Fang H. A comparison of blood vessels of the brain and peripheral blood vessels. In: Wright IS, Milikan CH: (EDS) Cerebral vascular diseases. New York: Grune \& Stratton, 1958:17-22.

21. Wilkinson IMS. The vertebral artery: extracranial and intracranial structure. Arch Neurol 1972;27:392-396.
22. Sinclair W. Dissecting aneurysm of the middle cerebral artery associated with migraine syndrome. Am J Pathol 1953;29:10-1083-1091.

23. Ringel SP, Harrison $\mathrm{SH}$, Norenberg MD, et al. Fibromuscular dysplasia: multiple 'spontaneous' dissecting aneurysm of the major cervical arteries. Ann Neurol 1977;1:301-304

24. Thapedi IM, Ashenhurst EM, Rozdilsky B. Spontaneous dissecting aneurysms of the internal carotid artery: in the neck. Arch Neurol 1970;23:549-554.

25. Austin MG, Schaefer RF. Marfan's syndrome with unusual blood vessel manifestations. Arch Pathol Lab Mad 1957;64:205-209.

26. Chang V, Rewcastle NB, Harwood-Nash DCF, et al. Bilateral dissecting aneurysms of the intracranial internal carotid arteries in an 8-year-old boy. Neurology 1974;25:573-579.

27. Sturzenegger M. Headache and neck pain: the warning symptoms of the vertebral artery dissection. Headache 1994;34:187-193.

28. Hinse $\mathrm{P}$, Thie A, Lachenmayer L. Dissection of the extracranial vertebral artery: report of four cases and review of the literature. J Neurol Neurosurg Psychiatry 1991;54:863-869.

29. Sekino H, Nakamura N, Kato Y, et al. Disecting aneurysms of the vertebrobasilar system: clincal and angiographic observation. No Shinkei Geka 1982;9:125-133.

30. Hunter J. The works of John Hunter In: JF Palmer (ed). Longman, Rees, Orme, Green, and Longman, London 1839;3:594-612. 